\title{
Mit Polyneuropathie Schnee geräumt
}

— Ein 70-jähriger Mann mit einer peripheren Neuropathie stellte sich mit dick angeschwollenen und schmerzhaften Fingern vor. Die Beschwerden waren aufgetreten, nachdem er bei Temperaturen unter $0^{\circ} \mathrm{C}$ für längere Zeit Schnee geräumt hatte, wobei er keine Handschuhe trug. Die Pulse waren tastbar und die Dopplerdruckmessung unauffällig. Der Mann wurde konservativ mit sterilen Verbänden, Hochlagerung der Arme und nicht steroidalen Antiphlogistika behandelt. Die mittlerweile aufgetretenen Blasen wurden eröffnet, um eine Hautschädigung durch die Anhäufung proinflammatorischer Prostaglandine und Thromboxane zu verringern. Es kam nicht zu einer dauerhaften Hautschädigung, woraus zu schließen war, dass der Patient zwar einen Kälte- schaden, nicht jedoch Erfrierungen erlitten hatte.

Alle Patienten mit bekannter peripherer Neuropathie, die sich bei kalten Temperaturen im Winter längere Zeit



im Freien aufhalten, sollten die Extremitäten durch geeignete Handschuhe bzw. Schuhwerk schützen. Das Kälteempfinden kann durch die Polyneuropathie zum Teil aufgehoben sein. Zusätzlich tragen trophische Störungen der Haut zu einer verstärkten Empfindlichkeit gegenüber Kälteschäden bei.

H. S. FÜESSL -

- C. Reichl und J. Bristol (Department of Surgery, Cheltenham General Hospital, Cheltenham GL53 7AN, UK) Brit. Med J. 343 (2011) d 6122

Kälteschaden durch Schneeräumen.

\section{ACHTUNG:}

\section{Hier muss der Dummy durch eine Anzeige ersetzt werden !!}

